\title{
Sensor Fusion Cognition using Belief Filtering for Tracking and Identification
}

\author{
Erik Blasch \\ AFRL/SNAT \\ 2241 Avionics Cir. \\ WPAFB, OH 45433 \\ blaschep@sensors.wpafb.af.mil
}

\author{
Lang Hong \\ Dept. of Electrical Engineering \\ Wright State University \\ Dayton, OH 45435 \\ lhong@cs.wright.edu
}

\begin{abstract}
Humans exhibit remarkable abilities to estimate, filter, predict, and fuse information in target tracking tasks. To improve track quality, we extend previous tracking approaches by investigating human cognitive-level fusion for constraining the set of plausible targets where the number of targets is not known a priori. The target track algorithm predicts a belief in the position and pose for a set of targets and an automatic target recognition algorithm uses the pose estimate to calculate an accumulated target-belief classification confidence measure. The human integrates the target track information and classification confidence measures to determine the number and identification of targets. This paper implements the cognitive belief filtering approach for sensor fusion and resolves target identity through a set-theory approach by determining a plausible set of targets being tracked.
\end{abstract}

Keywords: Sensor Fusion, Target Recognition, Classification, \& Identification, Tracking, Cognitive Belief Filter, HRR

\section{INTRODUCTION}

Humans and machines are typically trained for specific missions and/or scenarios. One such case is identification (ID) of a target. When the human approaches the target, either the target is moving, the human is moving, or both are moving. Cognition, the act of directing attention to sensory information, can be used by the human to fuse track and ID information as a perception of a set of moving targets. Dynamic cognitive multitarget-multisensor fusion under uncertainty requires target selection which can be formulated as a belief filtering problem in which sensed target states and identities are represented as current situational beliefs. The objective of the human is to 1) abstract number of tracks from the tracking environment, 2) assess confidence levels from the target classification algorithm, and 3) integrate the information for realtime beliefs of the number and types of targets from a plausible set of targets.

Multitarget tracking and ID is a subset of sensor fusion, which includes selecting sensors, sensor recognition policies, and tracking algorithms for a given set of mission requirements. For example, in a typical tactical aircraft, the onboard sensors are active radar, electro-optical, and navigation sensors, with each sensor having a variety of modes in which it can operate and features it can detect. Figure 1 shows the case of moving target indicator (MTI) and high range resolution (HRR) radar collection of range-bin features. The radar sensor makes kinematic measurements to detect, track, and classify objects of interest while reducing pilot workload. In a dynamic and uncertain environment, a sensor manager, such as a human, must fuse the track and classification information to ID the correct target at a given time and can aid tracking algorithms by determining a set of tracks to follow and aid classification algorithms by constraining the set of plausible targets.

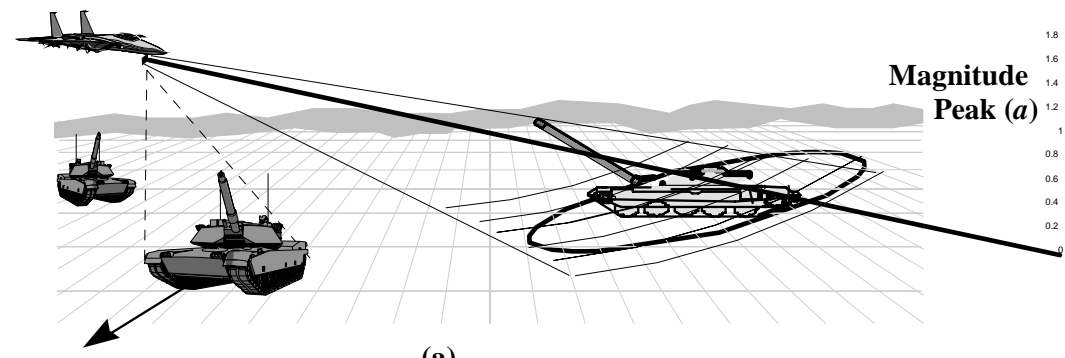

(a)

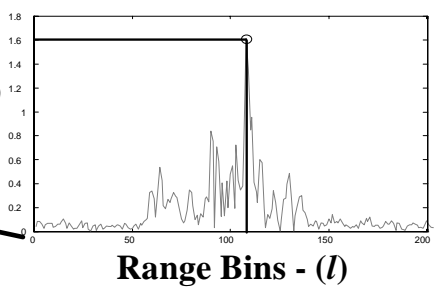

(b)

Figure 1. a) Multi-feature-Multitarget Air-to-ground HRR Target Tracking, and b) HRR Signature.

Multitarget tracking in the presence of clutter has been investigated through the use of data association algorithms [1]. Likewise, other multisensor fusion algorithms have focused on tracking targets from multiple look sequences[2]. One 
inherent limitation of current algorithms is that the number of targets needs to be known a priori. While tracking algorithms can speculate on the number of targets, the cognition of the number of targets can be afforded to the user. Once the human has an ID belief in the number of targets, the tracking algorithm can be updated.

A few tracking and identification algorithms have been proposed [3,4,5]. Layne [6] utilizes an automatic target recognition (ATR) and tracking filter in a multiple model estimator (MME) approach for HRR signatures. These approaches, although influential in this work, rely on the Bayes' rule for identification where the most probable target is selected. A limitation of using a Bayesian analysis is that it does not capture incomplete knowledge. For instance, there are times when unknown targets might be of interest that are not known at algorithm initiation. At other times, there are unknown number of targets to track or targets not trained for classification. We seek to expand on these tracking and ID algorithms for HRR signatures, by allowing for the capability to discern unknown relevant targets and reject non-plausible targets.

One way to study the problem is the interaction of the human and the machine working synergistically - since the sensors are extensions of the human's processing. The set theory approach to HRR target classification was proposed by Mitchell and Westerkamp [7,8] and termed a Statistical feature based classifier (STaF) and is influential in this work. In addition, Blasch [9] presented a feature-based set-theory approach for target tracking. In both cases, classification and tracking, a set of features and a set of targets was investigated. In the scenario investigated in the paper, we explore the use of belief filtering for a dynamic tracking environment, and by extending the STaF algorithm as a belief filter for HRR profiles, a plausible set of tracks and targets are made available to the user at each time instant. The human is presented both the tracking information and the accrued evidence for each target type as a confidence measure. The human must then cognitively determine from the set of targets how many and which target goes with which track. Additionally, the human has the ability to eliminate those targets that are not plausible which reduces the number of tracks and the set of pose templates from which the classification algorithm must search.

This paper develops a feature-based cognitive belief filter (CBF) for simultaneously tracking and identifying targets. The CBF algorithm leverages the belief filter evidence accrual tracking and ID [10] algorithm and the STaF algorithm [11]. By introducing the operator or image analyst for cognitive fusion, it may offer a means to control some aspects of the computational burdens experienced by analytical data association techniques while improving track quality for multitarget tracking and ID in the presence of clutter. Section 2 overviews the fusion levels and Section 3 describes the feature track and classification level fusion. Section 4 formulates the cognitive fusion problem. Section 5 presents results and Section 6 draws some conclusions.

\section{BELIEF FILTER FUSION FOR RADAR PROCESSING}

\subsection{Intelligent Feature and Track Level Fusion}

The ability to perform track and ID fusion requires fusion of kinematic information, fusion of HRR target features, and a higher level cognitive fusion of both the track and classification information. Like multitarget data association algorithms for accurately tracking targets in the presence of clutter, we assume that detected targets can be tracked from a sequence of center-of-gravity and pose positional data. Also, for a given sensor/target scenario, we assume detected HRR signature features can effectively be fused to discern target types with associated confidence measures. Feature-to-target mappings can be achieved either through training, learning, or predicted. By leveraging knowledge about target types from training, the target can be classified in real time. The fusion of classification and tracking information can significantly reduce processing time by selecting the number of target tracks to investigate as well as increase target track quality. In addition, correlating kinematic features with classified signatures will allow for identifying targets at the same time tracking is performed. Fusion of information takes place at either the 1) feature level for target recognition, 2) kinematic level for target tracking, or 3) cognitive level for target tracking and ID.

Cognition in tracking is the ability of an agent to detect patterns of tracks and use the confidence measures from the target classification results to determine a plausible set of targets for each track. Since confidence information available to an observer is restricted to a range of pose comparisons, the discernment of the number and type of targets is only a situational belief. However, as the belief evidence accumulates and the person reduces the plausible set of targets, target classification confidence will increase which can improve track quality and allow for a correct target ID. 


\subsection{High Range Resolution Radar}

Radar systems are effective for surveillance applications due to their distance range resolution invariance, all weather, and measurement capabilities. The radar antenna has a tradeoff between MTI, HRR, or SAR modes. HRR radar offers a method for imaging moving targets by extracting energy return from range profiles. If the target is stationary, a collection of HRR radar signatures can be processed to form a synthetic aperture radar (SAR) image. The concatenation of the HRR profiles to form a SAR image might take up to 10 seconds, which is lengthy in time-critical scenarios. A sensor mode that has recently received attention is HRR radar. In the HRR radar mode, the cross range resolution is the radar beam width, which is large at long range. Due to this lack of cross range resolution, HRR was not useful for stationary targets because clutter overwhelmed the target information. However, if the target is moving, it is possible to separate the target and clutter in Doppler [12]. Additionally, HRR processing can be interleaved with ground moving target indicator (GMTI) processing for detection. Once detected, tracking information can be used to derive pose angles for the targets. Tracking information enhances the target ID by reducing the number of unknown variables associated with the target. Likewise, target ID helps determine the number of target tracks. In addition to correctly identifying targets with HRR, a target ID system in military scenarios must also avoid incorrectly identifying unknown targets as known targets. Clearly, the ID system must be allowed to reject ID decisions rather than be forced to make a target ID. Requiring the system to maintain a high probability of correct ID while at the same time rejecting unknown targets is a very challenging problem. One way to combat the problem is by leveraging human cognitive fusion, which operates on accumulated beliefs and can coordinate HRR tracking and classification for correct target ID.

\section{FEATURE-BASED BELIEF TRACKING AND CLASSIFICATION}

One of the key links between HRR tracking and ID is the ability of the tracking filter to accurately position the ID sensor and estimate the target pose. Pose information consists of a depression angle and an aspect angle. The depression angle is related to the sensor position and aspect angle can be determined from the HRR profile. While Layne [6] uses the entire length of the HRR profile to determine the target, we utilize the peak features that are trained using the STaF classifier. Westerkamp and Mitchell [7] have shown that the peak features in a HRR map can accurately classify objects. Another deviation from the ATRF is that instead of just one HRR profile, we assume that we have multiple targets in the search space, much as in the case of a MTI plot. In order to determine which measurements are plausible, we utilize the belief filtering approach [10] which uses evidential belief updates to allow for processing unknown target information.

\subsection{Kinematic Tracking Belief Filter (TBF)}

MTI radar tracking assumes that after receiving the energy return from the radar, the approximate coarse position of the target results. Since a finite number of range bins are collected, the center bin is assumed to be the position of the target. Additionally, the radar collection has an associated depression and azimuth angle to the target. After resolving the direction of movement, the relative pose of the target is indicated for a belief classification to be performed.

The Tracking Belief Filter is an intelligent method which devotes attention to every believable measurement and cycles through kinematic features until a target position and pose is reached. The filter assumes the past is summarized by an approximate sufficient statistic - state estimates (approximate conditional mean) and covariances for each target. Each measurement

$$
\mathrm{z}(\mathrm{k})=\left[x_{t}(\mathrm{k}), \mathrm{f}_{1}, \ldots \mathrm{f}_{\mathrm{n}}\right]
$$

is sequenced as it comes in as depicted in Figure 2, and the kinematic state and ID feature variables are separated.

The target state and true measurement are assumed to evolve in time according to:

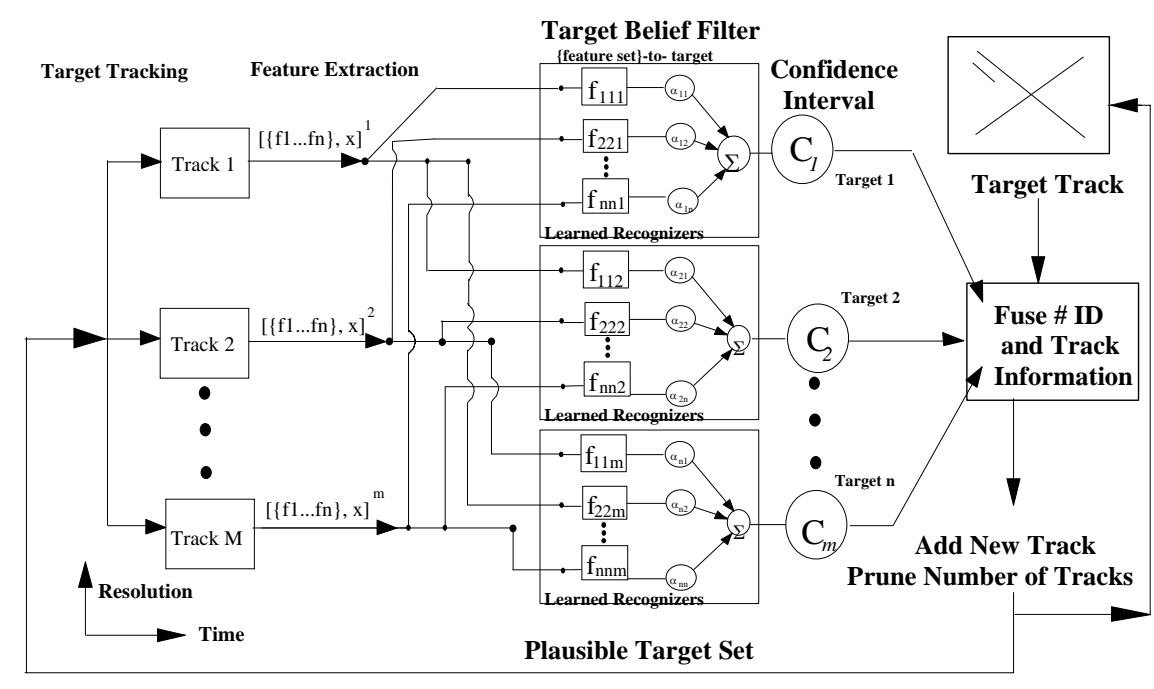

Figure 2. Feature-Recognition Tracking Model. 


$$
\begin{aligned}
& \mathrm{x}(k+1)=\mathrm{F}(k) \mathrm{x}(k)+\mathrm{v}(k) \\
& \mathrm{z}(k)=\mathrm{H}(k) \mathrm{x}(k)+\mathrm{w}(k)
\end{aligned}
$$

where $\mathrm{v}(k)$ and $\mathrm{w}(k)$ are zero-mean mutually independent white Gaussian noise sequences with known covariance matrices $\mathrm{Q}(k)$ and $\mathrm{R}(k)$, respectively. Spurious measurements are uniformly distributed in the measurement space. Tracks are assumed initialized at an initial state estimate $\mathrm{x}(0)$ for a plausible number of targets determined from the user.

The measurement-to-target association probabilities are computed across the targets and these probabilities are computed only for the latest set of measurements. The conditional probabilities of the joint-target association events pertaining to the current time $k$ is defined as $\theta(k)$, where $\theta_{j t}$ is the event that measurement $j$ originated from target $t, j=1, \ldots, m(k) ; t=0$, $1, \ldots, N_{t}$, where $m(k)$ is the total number of measurements for each time step, and $N_{t}$ is the known number of targets. A plausible elliptical validation region $V$ with a gate threshold, $\gamma$, is set up at every sampling time around the predicted measurement and is used to select believable measurements. Measurements from one target can fall in the validation region of the neighboring target and is persistent interference. All feature variables that carry information useful for discerning the correct measurement from the incorrect ones are assumed to be included in the measurement vector. The CBF approach differs from conventional algorithms in how kinematic measurements are used in the estimation of the kinematic state of the correct target. Figure 3 shows the tradeoffs that are determined from the kinematic level similar to the Probability Data Association Filter (PDAF)[1].

The plausible target validation matrix $\Omega=\left|\omega_{j t}\right|$ is composed of binary elements that indicate if measurement $j$ lies in the validation gate of target

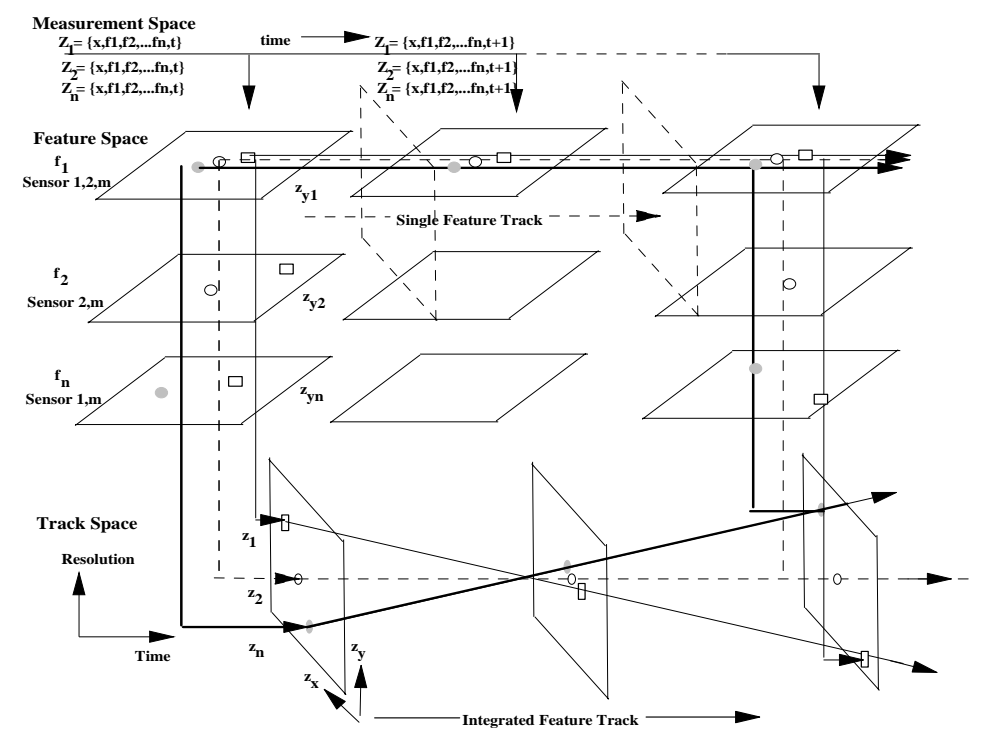

Figure 3. Kinematic Feature Tracking. $t$. The index $t=0$ stands for "none of the targets" and the corresponding column of $\Omega$ includes all measurements, since each measurement could have originated from clutter, false alarm, or the true target, or an unknown target. A joint association event consists of the values in $\Omega$ corresponding to the associations in $\theta$, assuming a single measurement source and at most one measurement originating from a target.

The believable joint association event probabilities are, using Bayes' Formula:

$$
\begin{aligned}
\mathrm{P}\left\{\theta(k) \mid \mathrm{Z}^{k}\right\} & =\mathrm{P}\left\{\theta(k) \mid \mathrm{Z}(k), \mathrm{m}(k), \mathrm{Z}^{k-1}\right\}=\frac{1}{\mathrm{C}} \mathrm{P}\left[\mathrm{Z}(k) \mid \theta(k), \mathrm{m}(k), \mathrm{Z}^{k-1}\right] \mathrm{P}\{\theta(k) \mid \mathrm{m}(k)\} \\
& =\frac{1}{\mathrm{C}} \prod_{\mathrm{j}=1}^{\mathrm{m}(k)-\phi(k)} \mathrm{V}\left\{\mathrm{f}_{t_{t}}(k)\left[\mathrm{z}_{j}(k)\right]\right\} \tau^{\tau_{j}}
\end{aligned}
$$

where $c$ is the normalization constant. From these probability values, the kinematic state $x_{j t}$ is updated and the pose information aspect angle is assessed as $\phi_{j \mathrm{t}}=\tan ^{-1}\left(y_{j \mathrm{t}}(k+1)-y_{j \mathrm{t}}(k) / x_{j \mathrm{t}}(k+1)-x_{j \mathrm{t}}(k)\right)$. The algorithm continues to track objects until the pose measurement is updated for a fixed time step $\Delta k$.

Note, from Figure 3, if only the kinematic feature information is used a data association error could result from closely spaced measurements in a time constrained decision making process. However, ID feature information, $f_{a l}$, can be fused together to associate the correct target type with the correct track. Thus, simultaneously associating target kinematic and classification features results in higher belief of true measurement-to-target value and reduces the target search. The TBF outputs a pose estimate, such as aspect angle, for each track given the sensor's azimuth and depression angle. By using pose, we can associate the position of the target with the aspect angle of the HRR sensor for feature target classification. 


\subsection{Feature-based Classification Belief Filter}

The belief classification algorithm is based on the statistical behavior of extracted features and is similar to the STaF classifier. The features, $f_{a l T}$, consist of, $a$, salient peak amplitudes, $l$, feature peak location, and $T$ the target profile. Target class hypotheses are defined as the set $\Omega=\left\{T_{1}, T_{2}, \ldots, T_{\mathrm{m}}\right\}$ for an algorithm trained on $m$ target classes. The peak location data are represented by $L=\left\{l_{1}, l_{2}, \ldots, l_{n}\right\}$ and the peak amplitude data by $A=\left\{a_{1}, a_{2}, \ldots, a_{n}\right\}$ for $n$ extracted peaks from an observed target signature. The extracted peak information (see Figure 1) from an observation is determined in real-time with priorities, $\alpha_{a l}$, being placed on the salient number of location features. The basic statistical modeling concept is to estimate the probability that a peak occurs in a specific location $l_{\mathrm{j}}$, given that the observation is from target $T_{\mathrm{i}}$, Further, the probability that the peak has amplitude $a_{\mathrm{j}}$ given that the peak is at the location $l_{\mathrm{j}}$ and that the observation is of target $T_{\mathrm{i}}$ must be determined. The primary estimated feature statistics required to determine these probabilities are the peak location probability function (PLPF) and the peak amplitude probability density function (PAPDF); see Mitchell[11] for more details.

The role of the Peak Location Probability Function (PLPF) estimation is to determine the probability that a peak will be observed in a specific range bin location given that the observation was from some individual target class $T_{\mathrm{i}}$. This probability is estimated from the peak locations of the training ensemble for each target class. A Parzen estimator with a normal kernel function along the range dimension is employed to estimate the PLPF[13]. With this function, class probabilities are associated with peak locations. However, this information alone is not enough to make robust target classification. The additional information will come from the conditional peak amplitude statistics.

The Peak Amplitude Probabilities Density Function (PAPDF) uses amplitude statistics which are conditional on the occurrence of a peak in a specific location and for a given target class. This estimation approach ensures that the amplitude statistics are based only on the detected features rather than a specific range bin location. The form of the amplitude statistical distribution is assumed to be Normal within a given range bin. While it is known that the magnitude of the signatures has a Rician distribution, the Gaussian assumption is reasonable if a "power transform" is performed [11]. The transformation simplifies the problem since the normal PDF is completely specified by two parameters, the mean and variance. These parameters are calculated for each range bin from the amplitudes of the extracted peaks of the training ensemble.

Given the PLPF and peak amplitude PDFs, class likelihoods and probabilities must be calculated for a set of features. The feature location likelihoods are found by evaluating the PLPF at a specific feature location. The amplitude likelihoods are found in a similar way using a mathematical expression for the Gaussian PDF. The parameters of the Gaussian PDF are the estimated mean and variance terms. The likelihood that the observed feature amplitude is the result of observing target class $T_{\mathrm{i}}$ is found by evaluating

$$
p\left(a_{\mathrm{j}} \mid T_{\mathrm{i}} l_{\mathrm{i}}\right)_{\phi}=\left[1 / \sqrt{2 \pi \sigma_{\mathrm{ij}}}\right] \exp \left[-\frac{\left(a_{\mathrm{j}}-\mu_{\mathrm{ij}}\right)^{2}}{2 \sigma_{\mathrm{ij}}}\right]
$$

where $\mu_{\mathrm{ij}}$ and $\sigma_{\mathrm{ij}}$ are the conditional mean and standard deviation for peak location $l_{\mathrm{j}}$ given class $T_{\mathrm{i}}$ and target aspect angle $\phi$. Note that this likelihood is conditioned on both the target class and the feature location. The joint peak location and amplitude likelihood is calculated by multiplying the individual likelihoods,

$$
p\left(l_{\mathrm{j}} a_{\mathrm{j}} \mid T_{\mathrm{i}}\right)_{\phi}=p\left(a_{\mathrm{j}} \mid T_{\mathrm{i}} l_{\mathrm{i}}\right)_{\phi} P\left(l_{\mathrm{j}} \mid T_{\mathrm{i}}\right)_{\phi}
$$

From the joint likelihoods a posteriori probabilities are calculated using Bayes rule,

$$
P\left(T_{\mathrm{i}} \mid l_{\mathrm{j}} a_{\mathrm{j}}\right)_{\phi}=\frac{p\left(a_{\mathrm{j}} l_{\mathrm{j}} \mid T_{\mathrm{i}}\right)_{\phi} P\left(T_{\mathrm{i}}\right)}{\sum_{\mathrm{q}=1}^{\mathrm{m}} p\left(a_{\mathrm{j}} l_{\mathrm{j}} \mid T_{\mathrm{q}}\right) P\left(T_{\mathrm{q}}\right)}
$$

One problem associated with the Bayesian probability calculations is that only relative probabilistic information is considered rather than the set of global information. Bayes rule normalizes the probabilities relative to the likelihoods of some set of target hypotheses. Therefore, only probabilistic information about a class relative to the probabilities of some set of other classes is given. Consider the case that an observation is from a target for which no statistics are available. If that observation looks statistically more like one class, say class $T_{1}$, than the other classes, the Bayesian probabilities would appear to be confident that the observation belonged to class $T_{1}$. In reality the likelihood, $p\left(a_{\mathrm{j}} l_{\mathrm{j}} \mid T_{1}\right)_{\phi}$, may be very 
low, resulting in a decision error. Therefore, if it is possible that unknown targets will be observed, then Bayes decision alone will not be able to reject incorrect decisions due to the unknown target. The information required to eliminate these errors can be obtained from the likelihood values by using a confidence interval.

\subsubsection{Confidence Value Calculation}

The inclusion of likelihood information in this algorithm will be in the form of a confidence measure. The next sections will discuss the determination of the confidence, how the individual peak confidence levels and $a$ posteriori probabilities are used to determine class beliefs, and how to accrue the class beliefs to obtain an overall track classification decision.

The most complicating requirement with HRR classification of ground target is the rejection of unknown target classes. This problem occurs because it is impossible to train a classifier to recognize every possible ground target. Therefore, the belief classification algorithm must only make classification decisions when the statistical confidence is high. The decision confidence measures are based on the class likelihoods using PDFs developed for each class. The likelihood statistics are obtained by comparing the training exemplars with their own statistical model.

The actual likelihood PDFs are estimated using a Parzen estimator with a normal kernel function. Observing that the likelihoods are class-conditional probabilities, larger likelihood decisions should have a higher confidence level. A function that mirrors this concept is the cumulative distribution

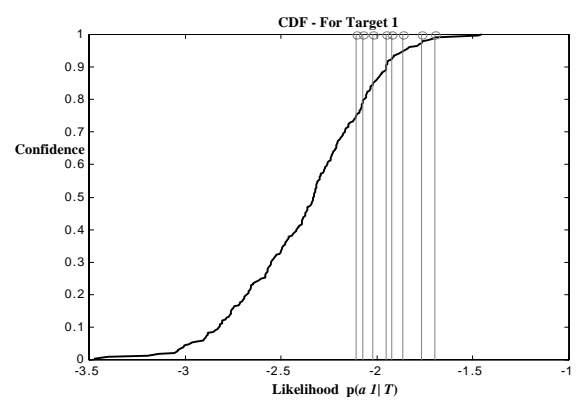

Figure 4. In-Class likelihood CDF used to determine decision confidence. function (CDF). For this reason, the CDF of in-class likelihood PDFs is used to determine the decision confidence. An example of a likelihood CDF is shown in Figure 4. At any likelihood $x$ the CDF evaluates the probability $P\left(p\left(a, l \mid T_{\mathrm{i}}\right)_{\phi} \leq x\right)$, which represents the decision confidence. Note that both the likelihoods and the CDFs are probabilities and therefore their values are in the range [0,1]. Additionally, $P\left(p\left(a, l \mid T_{\mathrm{i}}\right)_{\phi}\right) \leq 0.0=0$ and $P\left(p\left(a, l \mid T_{\mathrm{i}}\right)_{\phi}\right) \leq 1.0=1$ represent the minimum and maximum confidence values.

To obtain the confidence measure, a target class hypothesis, $T_{\text {hyp }}$, must be made. It is assumed that the observation belongs to class $T_{\text {hyp }}$, resulting in the in-class likelihood $p\left(a_{\mathrm{j}} l_{\mathrm{j}} \mid T_{\mathrm{hyp}}\right)_{\phi}$. The $T_{\text {hyp }}$ decision confidence for peak $j$ is calculated by evaluating the likelihood CDF at $p\left(a_{\mathrm{j}} l_{\mathrm{j}} \mid T_{\mathrm{hyp}}\right)_{\phi}$ which represents the confidence that the observed peak is associated with target class hypothesis $T_{\text {hyp }}$. Therefore, each target hypothesis of interest must have an associated confidence value for each of the extracted peaks in an observation. Typically this corresponds to calculating a confidence value for each of the $m$ target classes, $T_{\mathrm{i}}$.

\subsubsection{Belief-Hypothesis Confirmation}

The classification belief filter simulates the confirmation process people perform by predicting hypotheses in a frame of discernment, $\Theta$. The frame of discernment consists of a collection of matched features, $\Theta=\cup\left\{f_{1}, \ldots, f_{n}\right\}$. Only a subset of the entire combinations of features is possible. Thus, the belief set is a modification of Shafer's belief functions to only include a priori trained set of feature combinations. The probabilistic fusion of extracted features is performed using Dempster's rule. Dempster's rule is modified to assign a priority, $\alpha$, to salient features and discounted over time, $\gamma$, to reflect a change in feature saliency from previous signature classifications. For individual peak features, class likelihoods, a posteriori probabilities, and decision confidences from joint likelihoods are calculated. These statistics are used to develop a set of beliefs for specific target hypotheses. The confidences weight the class a posteriori probabilities to create a belief in a specific target class. The beliefs are found using

$$
b_{\mathrm{k}}^{T_{\text {hyp }}}\left(T_{\mathrm{i}} \mid a_{\mathrm{j}} l_{\mathrm{j}}\right)_{\phi}=C_{T_{\text {hyp }}}^{j}(k) P\left(T_{\mathrm{i}} \mid a_{\mathrm{j}} l_{\mathrm{j}}\right) \gamma(k),
$$

where $C_{T_{\text {hyp }}}^{j}(k)$, is the confidence that the $\mathrm{j}^{\text {th }}$ peak is associated with the target hypothesis $T_{\text {hyp }}$ for time $k$ and $\gamma(k)$ is a timediscounting effect in evidence accumulation. Since the confidence is based on a class hypothesis, the beliefs generate a matrix, each column of which is associated with a particular class hypothesis. Additionally, an uncertainty value is calculated as $U_{T_{\text {hyp }}}^{j}(k)=1-C_{T_{\text {hyp }}}^{j}(k)$ and appended to the bottom of belief hypothesis matrix. Note that since the sum of the 
a posteriori probabilities is unity, the sum of the beliefs and uncertainty for any given hypothesis is also unity, $U_{T_{\text {hyp }}}^{j}(k)+\sum_{\mathrm{i}=1}^{\mathrm{n}} b_{\mathrm{k}}^{T_{\text {hyp }}}\left(T_{\mathrm{i}} \mid a_{\mathrm{j}} l_{\mathrm{j}}\right)_{\phi}=1$.

The generation of the beliefs and uncertainties directly ties the confidence that an observed feature is associated with a target hypothesis. Thus, a high uncertainty occurs when the likelihood that the observed feature is not associated with the hypothesized target. By using uncertainty information, unknown target observations are resolved and can be used to determine if the set of targets needs to be increased. Robustness and confidence are obtained through evidential fusion of the individual feature decisions.

\subsubsection{Peak Feature Belief Evidence Accrual}

For each feature extracted from an observation a belief hypothesis matrix is generated. Each matrix contains evidence that is accrued to aid in the acceptance or rejection of each target class hypothesis. The individual feature beliefs are fused using Dempster's rule of combination [14]. The fusion rule allows for decision uncertainty and confidence values to be assigned to classification decisions. The uncertainty between features 1 and 2 for a target hypothesis is:

$$
U^{1 ; 2}(k)=\frac{U^{1}(k) U^{2}(k)}{1-\sum_{j=1}^{\mathrm{n}} \sum_{\substack{\mathrm{n} \\ k=1 ; k \neq \mathrm{j}}} b_{\mathrm{k}}\left(a_{1} l_{1}\right) b_{\mathrm{k}}\left(a_{2} l_{2}\right)}
$$

where the denominator normalizes the beliefs. The fused uncertainty can be updated with the new beliefs by

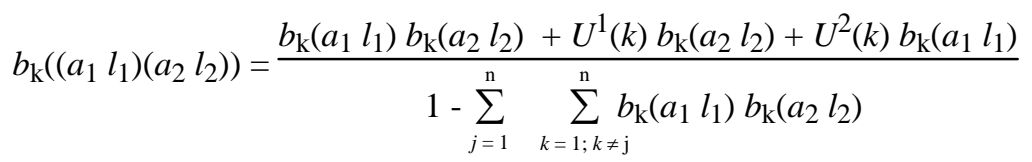

These belief equations are recursively applied to the entire set of extracted features to calculate the overall class beliefs and uncertainties for a specific class hypothesis given the pose estimation from the tracking algorithm. Additionally, the last element of each belief matrix column is the fused uncertainty associated with a specific target class hypothesis. The target classification confidence is made by selecting the largest belief on the diagonal of the matrix and is presented to the user for cognitive decision making.

\subsection{Cognitive Level Fusion -ID Belief Filter}

Using the fusion techniques described above, one obtains a target class belief and confidence for all plausible targets for each track. The decision uncertainty and confidence are used to determine the quality of the target classification and reject unknown spurious target track observations. The technique statistically models the uncertainty associated with a correct target class hypothesis for a given target from an estimated pose. These data are generated by using in-class uncertainty statistics determined from the training ensemble. These "in-class" statistics are the uncertainties associated with classification of target class $T_{\mathrm{i}}$ under the hypothesis that the target is from class $T_{\mathrm{i}}$. The "in-class" information is accrued in the D-S algorithm for each time step, allowing the fused decision confidence to be assessed. The evidence accrual becomes important when one needs to reject unknown target observations. Cognitive level fusion includes not only rejection, but the selection of plausible targets for each track. Additionally, by accruing evidence of feature space, through classification, and time, through tracking, evidence can be fused for target ID. The determination of the efficient target identity is the result of cognitive fusion from the human. Once a plausible ID is made for a set of targets, the fused information updates the track information and recalculates the confidence measures. Recursively, target sets are reduced and tracks are continued.

\subsection{Fused Track and Classified State Estimation}

Assuming the targets conditioned on the past observations are mutually independent, the decoupled state estimation (filtering) of the marginal association probabilities, which are obtained from the joint feature probabilities of track pose and target belief, is obtained by summing over all joint events in which the marginal event of interest occurs. The conditional probability of the event is:

$$
\beta_{j t}(k) \stackrel{\Delta}{=} \mathrm{P}\left\{\theta_{j t}(k) \mid \mathrm{Z}^{k}\right\} \cdot b_{\mathrm{k}}^{T_{\text {hyp }}}\left(T_{\mathrm{i}} \mid a_{\mathrm{j}} l_{\mathrm{j}}\right)_{\phi}
$$




$$
=\sum_{\theta} \mathrm{P}\left\{\theta \mid \mathrm{Z}^{k}\right\} \hat{\omega}_{j t}(\theta) \cdot b_{\mathrm{k}}^{T_{\text {hyp }}}\left(T_{\mathrm{i}} \mid a_{\mathrm{j}} l_{\mathrm{j}}\right)_{\phi}=\sum_{\theta: \theta_{\mathrm{jt}} \in \theta} \mathrm{P}\left\{\theta \mid \mathrm{Z}^{k}\right\} \cdot b_{\mathrm{k}}^{T_{\text {hyp }}}\left(T_{\mathrm{i}} \mid a_{\mathrm{j}} l_{\mathrm{j}}\right)_{\phi}
$$

The algorithm decomposes the estimation with respect to the origin of each element of the latest set of validated measurements. Using the total probability theorem, with respect to the above events, the conditional mean of a target state at time $k$ can be written as:

$$
\hat{\mathrm{x}}(k \mid k)=\sum_{\mathrm{i}=0}^{m(k)} \hat{\mathrm{x}}_{\mathrm{i}}(k \mid k) \beta_{\mathrm{i}}(k)
$$

where $\mathrm{x}(k \mid k)$ is the update state conditioned on the event that the $i^{\text {th }}$ validated measurement is correct.

The state estimate, conditioned on measurement $i$, is:

$$
\begin{aligned}
& \hat{\mathrm{x}}_{\mathrm{i}}(k \mid k)=\hat{\mathrm{X}}_{\mathrm{i}}(k \mid k-1)+\mathrm{W}(k) v_{\mathrm{i}}(k) \\
& v_{\mathrm{i}}(k)=\mathrm{z}_{\mathrm{i}}(k)-\hat{\mathrm{z}}(k \mid k-1) \\
& \mathrm{W}(k)=\mathrm{P}(k \mid k-1) \mathrm{H}(\mathrm{k})^{\mathrm{T}} \mathrm{S}(k)^{-1}
\end{aligned}
$$

The combined state update equation, combined innovation, and covariance associated with the state are:

$$
\begin{aligned}
& \hat{\mathrm{x}}(k \mid k)=\hat{\mathrm{x}}(k \mid k-1)+\mathrm{W}(k) v(k) \\
& v(k)=\sum_{\mathrm{i}=1}^{m(k)} \beta_{\mathrm{i}}(k) v_{\mathrm{i}}(k) \\
& \mathrm{P}(k \mid k)=\beta_{\mathrm{o}}(k) \mathrm{P}(k \mid k-1)+\left[1-\beta_{\mathrm{o}}(k)\right] \mathrm{P}^{\mathrm{C}}(k \mid k)
\end{aligned}
$$

\section{PROBLEM FORMULATION}

Consider Figure 1 as an environment that the a pilot is monitoring. By assumption, the aircraft carries a HRR sensor able to detect target signatures from $T$ targets. For each target, an HRR profile is measured from a given pose angle and contains peak measurement features. Dynamic target measurements $z$ are taken at time steps $k$, which include target kinematics and classification peak features $\mathrm{z}(\mathrm{k})=\left[x_{t}(\mathrm{k}), \mathrm{f}_{1}, \ldots \mathrm{f}_{\mathrm{n}}\right]$. The probability density of each measurement depends on whether the target is actually present or not. Further assume that a fixed number of kinematic and feature measurements will be taken at each time interval, where we model the clutter composing spurious measurements. A final decision form the CBF algorithm is rendered as to which target-type is associated with the $[x, y]$ measurement.

Three feature level fusion methods are implemented. The first, which we call Measurement Tracking, searches through all the measurements and probabilistically chooses the measurements most likely to be associated with the target and calculates the pose estimate. The second method, Feature Classification, is a procedure that uses feature measurements for believable target classes to discriminate between targets. The Cognitive Fusion level chooses from the set of classified targets a plausible set for each track and accumulates evidential classifications for target ID. Six targets were trained as to the feature locations of the HRR profiles and five were left unknown, as shown in Table 1. After the features were extracted and trained an online scenario was used to assist in the tracking and ID of targets. Initially, cluttered measurements were produced and the HRR profiles associated with each of the cluttered measurements. The cluttered measurements for each track consisted of ten positional points where an unknown number of targets were initiated.

Table 1. MSTAR Target Designations

\begin{tabular}{|c|c|}
\hline Known Targets & Unknown Targets \\
\hline \hline bmp2_apc & hmmwv_jeep \\
\hline btr70_transport & m110_gun \\
\hline m1_tank & m113_transport \\
\hline m2_apc & m35_truck \\
\hline m109_gun & m548_tank \\
\hline t72_tank & \\
\hline
\end{tabular}

The HRR data set used in this paper is derived from the public Moving and Stationary Target Acquisition and Recognition (MSTAR) program database. The MSTAR data consists of synthetic aperture radar (SAR) data at X-band $1 \times 1$ foot resolution. Images were recorded at $15^{\circ}$ and $17^{\circ}$ depression angles with full aspect $360^{\circ}$ coverage, at approximately one degree spacing in azimuth. The methodology used to convert the SAR imagery to HRR is discussed in [15]. While the MSTAR data consists of all aspect data, for each measurement pose estimate, viewing aspects considered are $-5^{\circ}<\phi<5^{\circ}$ in azimuth and $15^{\circ}$ and $17^{\circ}$ depression. Each of the SAR images results in 101 HRR profiles, which represent approximately a $3^{\circ}$ variation in azimuth. 
At the start of the algorithm, the cluttered track measurements were computed for each step and assigned a target type. The algorithm stops after ten measurements and determines the pose estimate for each track. Each pose is then used to extract a HRR profile to be used as the classification against the true trained pose HRR pose set. A confidence of classification is computed as to the belief in the target for each track. From the classification algorithm, the user, looks at the accumulated belief and the associated confidence interval to determine a plausible set of targets. By selecting the most plausible targets, the tracking algorithm was updated with a new number of targets. If more tracks were needed the algorithm would then start new tracks in addition to the ones that were previously visible. If a fewer number of tracks were needed, the algorithm would prune tracks to the ones that were most plausible. Another 10 measurements were conducted until a finite number of tracks and targets resulted.

\section{SIMULATION RESULTS}

The CFB track and ID method is evaluated with a Monte Carlo simulation and the performance metric is the final accumulated target ID belief. As detailed in Figure 5 by the true trajectory, the targets 1 ) start with position and velocity, 2) pass by each other at a close distance, and 3) finish with a specified pose. From the figures below (see Figure 6 and 7), by assuming the incorrect number of targets can lead to an incorrect target tracking result. However, if the user cognitively determines that the number of targets is not correct, then he has the option to alter the number of targets. In Figure 8, one of many situations run, the user correctly identifies the target track.

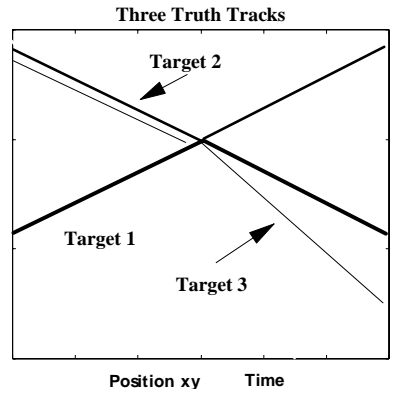

Figure 5. Truth Target Tracks.

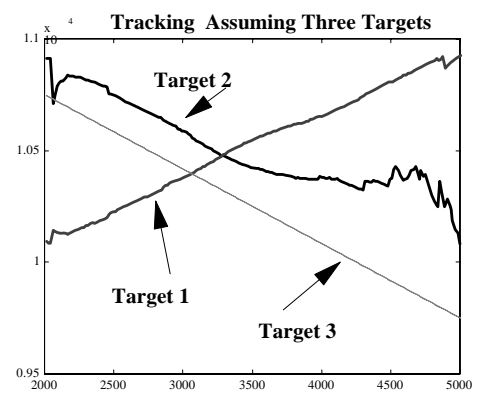

Figure 6. 3 Targets.

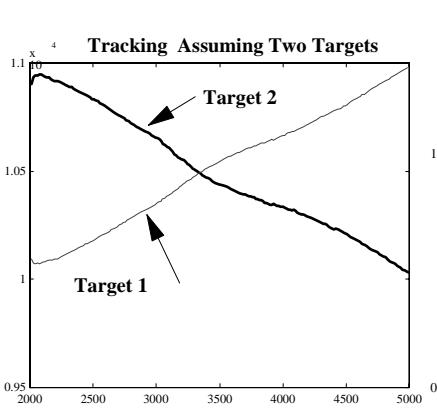

Figure 7. 2 Targets.

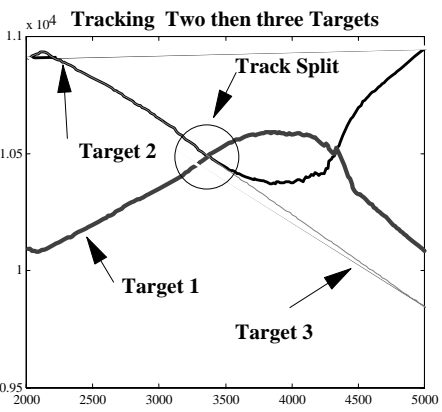

Figure 8. Start 2 Targets,

At the end of the run, we can see from Figures 9 through 10 which display the human computer interface, that the correct target is identified (lower right). However, at the beginning of the simulation, the tracker and identifier had no information

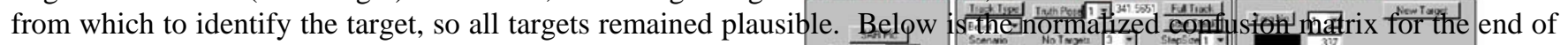
the run:

Table 2. Confusion Matrix for three targets.

\begin{tabular}{|c|c|c|c|c|c|c|c|}
\hline \multicolumn{2}{|l|}{ Target } & \multicolumn{2}{c|}{ Pose } & \multicolumn{5}{c|}{ Confusion } & Matrix \\
\hline Belief & Actual & Belief & True & BMP & BTR & HMV & M10 \\
\hline 1 & BMP & 24 & 18 & $\mathbf{0 . 9 6 5}$ & 0.415 & 0.625 & 0.268 \\
\hline 2 & BTR & 337 & 341 & 0.544 & $\mathbf{0 . 9 9 2}$ & 0.682 & 0.563 \\
\hline 3 & M109 & 328 & 320 & 0.662 & 0.410 & 0.758 & $\mathbf{0 . 7 8}$ \\
\hline
\end{tabular}

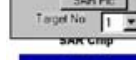

Table 2 indicates the usefulness of providing a cognitive interface

\section{CONCLUSIONS}

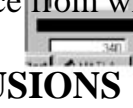

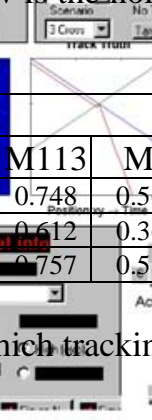

Figure 9. Assessment of Target 1.

Conventional measurement tracking techniques have difficulty in identifying targets when the number of targets is not known a priori. This paper has presented a cognitive belief filter tracker and applied it to the problem of HRR air-toground moving target identification. This research included training a belief classifier and integrating it into a tracking simulation with real data. The classifier is statistically based and operatesing features that are extracted from the

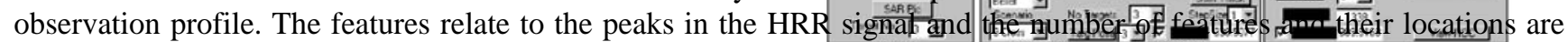
variable from observation to observation, depending on the signatinformation. In a series of simulaftorit" experiments, the CBF algorithm, which uses the feature measurements, identifies tracks at a faster rate than conventional multitarget-multisen demonstrates promise for multitarget tracking problems and war

Erik Blasch and Lang Hong

t targets and assigns measurements $\mathrm{to}^{\text {the the }}$ target ing methodologies. The presentedtectnique er exploration in problents where environmental
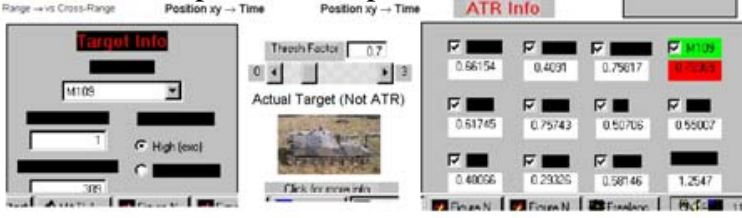

Figure 10. Assessment of Target 3. 
effects, occlusions, lost sensor data, and unknown targets can be cognitively assessed by humans that are not readily handled by current tracking and ID algorithms.

\section{ACKNOWLEDGEMENTS}

The first author would like to thank the Math and Geosciences Directorate of AFOSR for a grant 2312R1 that supported this research. Additionally, we would like to thank Jeff Layne, John Westerkamp, and Rick Mitchell for their helpful comments.

\section{REFERENCES}

[1] Y. Bar-Shalom and X. Li, Multitarget-Multisensor Tracking: Principles and Techniques, YBS, New York, 1995.

[2] Z. Ding and L. Hong, "Decoupling probabilistic data association algorithm for multiplatform multisensor tracking," Optical Engineering, ISSN 0091-3286, Vol. 37, No. 2, Feb. 1998.

[3] K. Kastella, "Joint multitarget probabilities for detection and tracking," SPIE AeroSense '97, April 21-25, 1997.

[4] M. Efe and D. Atherton, "A Tracking Algorithm for both Highly Maneuvering and Non-maneuvering Targets," CDC '96, San Diego, CA, 1997, pg. 3150 - 3155.

[5] E. Libby, "Application of sequence comparison methods to multisensor data fusion and target recognition," Ph.D. Dissertation, AFIT, June 1993.

[6] J. Layne, “Automatic Target Recognition and Tracking Filter,” SPIE AeroSense - Small Targets, April 1998.

[7] R. Mitchell and J. Westerkamp, "Statistical Feature Based Target Recognition,” NAECON, 1998, pp. 111-118.

[8] R. Mitchell and J. Westerkamp, "High range resolution radar target identification using a statistical feature based classifier with feature level fusion," in ATRWG, Huntsville, AL, October 1997.

[9] E. Blasch and J. Gainey, "Feature Based Biological Sensor Fusion,” Intl. Conference on Info. Fusion, 1998, pp. 702709.

[10]E. Blasch and L. Hong, "Simultaneous Tracking and Identification," Conference on Decision Control, Tampa, FL, December 1998, pg. 249-256.

[11] R. Mitchell. Robust High Range Resolution Radar Target Identification using a Statistical Feature Based Classifier with Feature Level Fusion. PhD thesis, University of Dayton, Dayton, OH, December 1997.

[12] M. Fennell and R. Wishner, "Battlefield awareness via synergistic SAR and MTI exploitation," IEEE Aerospace and Electronic Systems Magazine, February 1998.

[13] K. Fukunaga, Introduction to Statistical Pattern Recognition. Academic Press, Inc., second edition, 1990.

[14]E. Waltz and J. Llinas, Multisensor Data Fusion, Artech House, 1990.

[15] J. Westerkamp, S. Worrell, R. Williams, D. Wardell, and M. Ressler, "Robustness issues for id air-to-ground moving target ATR,” in Automatic Target Recognition Working Group, Huntsville, AL, October 1997. 